\title{
Long-term Outcome of the Retrourethral Transobturator Male Sling After Transurethral Resection of the Prostate
}

\author{
Alexander Kretschmer ${ }^{1,2}$, Alexander Buchner ${ }^{1}$, Benedikt Leitl ${ }^{1}$, Markus Grabbert ${ }^{1}$, Anne Sommer ${ }^{1}$, Wael Khoder ${ }^{3}$, \\ Christian Gozzi ${ }^{4}$, Christian G. Stief ${ }^{1}$, Ricarda M. Bauer ${ }^{1}$ \\ ${ }^{1}$ Department of Urology, Ludwig-Maximilians-University, Munich, Germany \\ ${ }^{2}$ Department of Urological Sciences, Vancouver Prostate Centre, University of British Columbia, Vancouver, Canada \\ ${ }^{3}$ Department of Urology, Johann-Wolfgang Goethe University, Frankfurt, Germany \\ ${ }^{4}$ Department of Urology, Marienklinik, Bolzano, Italy
}

\begin{abstract}
Purpose: To evaluate long-term outcomes of AdVance and AdVanceXP male slings in patients with persistent stress urinary incontinence (SUI) after transurethral resection of the prostate (TURP).

Methods: A total of 18 consecutive patients received AdVance $(n=14)$ or AdVanceXP $(n=4)$ male sling implantation between 2007 and 2013. Continence was determined by pad use, 24-hour pad testing and validated questionnaires (International Consultation on Incontinence Questionnaire Short Form, ICIQ-SF). Quality of life was evaluated by International Quality of Life (IQoL) score. Patient satisfaction was measured with patient's global impression of improvement score. Cure was defined as $0-5 \mathrm{~g}$ in the 24-hour pad test. Statistical analysis included Fisher exact and Wilcoxon test $(\mathrm{P}<0.05)$.

Results: Follow-up was available for 15 patients who underwent further analysis. After a median follow-up of 70 months (range, 18-83 months), mean daily pad usage was $1.8 \pm 2.1$ pads ( $\mathrm{P}=0.015$ vs. baseline level). Mean IQoL score was $66.4 \pm 31.6$ ( $\mathrm{P}=0.050$ vs. baseline level), and mean ICIQ-SF score was $9.5 \pm 6.6(\mathrm{P}=0.077$ vs. baseline level). Based on 24-hour pad testing, mean daily urine loss was $31.2 \pm 64.5 \mathrm{~g}$ (median, $0 \mathrm{~g}$; range, $0-209 \mathrm{~g}$ ). Cure rate was $46.7 \%$, and cure-and-improved rate was $60.0 \%$. Assessing predictive features for success, better results were found in patients who needed up to 4 pads preoperatively $(\mathrm{P}=0.041)$ as well as for patients $\leq 71$ years at the time of implantation $(\mathrm{P}=0.041)$.

Conclusions: The findings indicate that AdVance and AdVanceXP implantation can be performed effectively and safely in men suffering from SUI after TURP. However, long-term success rates seem to be lower compared to SUI after radical prostatectomy and patients should be counseled accordingly.
\end{abstract}

Keywords: Urinary Stress, Incontinence; Suburethral Slings; Transurethral Resection of the Prostate; Complications

- Research Ethics: The study was performed according to the Helsinki Declaration and approved by the Institutional Review of the Department of Urology, Ludwig-Maximilians University, Munich, Germany, where the experiment was performed. A written informed consent was obtained from all subjects.

- Conflict of Interest: R. M. Bauer declares consultancy work, lectures, and participation in clinical trials for AMS (Minnetonka, MN, USA) and Promedon (Cordoba, Argentina). C. Gozzi is the Co-founder of the AdVance male sling. The remaining authors have nothing to disclose.

\section{INTRODUCTION}

When conservative management of male stress urinary incon- tinence (SUI) fails, a surgical approach is recommended [1]. Male slings are currently of emerging interest and patient demands are increasing [2]. While there is strong evidence for the
Corresponding author: Alexander Kretschmer (iD http://orcid.org/0000-0002-6511-4354 Department of Urology, Ludwig-Maximilians-University, Marchioninistrasse 15 81377 Munich, Germany

E-mail: Alexander.kretschmer@med.uni-muenchen.de / Tel: +49-89-4400-0 / Fax: +49-89-4400-5444

Submitted: May 28, 2016 / Accepted after revision: June 18, 2016 
use of retrourethral transobturator male slings after radical prostatectomy, there is still very limited information available about their efficacy after transurethral resection of the prostate (TURP).

There is increasing evidence favoring the use of retrourethral transobturator male slings in the treatment of mild-to-moderate post-prostatectomy incontinence [3]. However, most of the single-center and multicenter studies excluded patients who had previously undergone TURP [4-8]. Rehder et al. [9] as well as Cornu et al. [10] included 9 and 5 patients respectively with SUI after TURP but did not report a specific subgroup analysis. In 2010, Wadie [11] reported the 2-year outcome of a retropubic bulbourethral male sling that was designed at Mansoura University and is similar to the AdVance male sling. The authors included 17 patients after TURP in their analysis without reporting particular outcomes for this subgroup. Thus, evidence for the treatment of TURP-derived SUI is urgently needed [12]. Despite the lack of evidence, based on current expert opinions, male slings are seen as potential therapeutic options in patients with SUI after TURP if preoperative diagnostics do not show any contraindications $[3,13]$.

Based on the current literature, $2.2 \%$ of patients undergoing TURP suffer from persistent SUI [12]. However, it seems likely that this represents only a conservative estimate and true SUI rates may be even higher. To address the lack of evidence regarding an increasing patient collective, we performed a crosssectional study and present results of a case series of 18 consecutive patients that underwent implantation of an AdVance or AdVanceXP male sling in one high-volume institution.

\section{MATERIALS AND METHODS}

\section{Patient Population, Inclusion, and Exclusion Criteria}

Between 2007 and 2013, more than 400 AdVance and AdVanceXP male sling implantations (Boston Scientific, Marlborough, USA, formerly American Medical Systems, Minnetonka, MN, USA) have been performed by 2 surgeons (A, B) - over 200 each - in one tertiary care center. The main cause for male sling implantation was postprostatectomy incontinence (PPI). After approval by the Institutional Review Board, 18 consecutive patients who received AdVance $(\mathrm{n}=14)$ or AdVanceXP $(\mathrm{n}=4)$ male sling implantation between 2007 and 2013 due to persistent SUI after TURP were included in the current study. Patients with previous invasive incontinence therapy, urethroscopically confirmed defect of the external urethral sphincter, and patients with previous pelvic radiotherapy were excluded from the current study. There were no patients who underwent previous bulking agent therapy.

\section{Surgical Technique, Study Design, and Data Assessment}

Urethroscopy, including a repositioning test, was performed preoperatively in every case as previously described [14]. Urodynamics were performed whenever concomitant urgency symptoms were stated. The AdVance and AdVanceXP implantations were performed by the 2 surgeons previously mentioned [15].

To analyze pre- and perioperative patient characteristics, patient charts were evaluated. In August 2015, a standardized questionnaire was sent to all participants. Hereby, the following validated tools were assessed: International Consultation on Incontinence Questionnaire Short Form (ICIQ-SF) [16], International Quality of Life (IQoL) score [17], and patient's global impression of improvement (PGI) [18], and a visual analogue scale (VAS) for the inguinal as well as perineal region. Additionally, the questionnaire included detailed instructions for a 24-hour pad test and enough pads for more than 24 hours. These instructions are well established at the institution and have been used in multiple previous studies $[19,20]$. Daily pad usage was assessed. Additionally, potential complications were recorded using the Clavien-Dindo classification [21].

Success ( $=$ cure) was defined as $\leq 5 \mathrm{~g}$ in the 24 -hour pad test. Improvement was defined as $>50 \%$ reduction in daily pad use after 24 hours.

\section{Statistical Analysis}

Primary endpoint was the success rate. To assess the changes in outcome at baseline level and after follow-up, the Wilcoxon signed rank test was used. To determine the impact of various potential associative factors on postoperative success rate, Fisher exact test was used for categorical parameters and the MannWhitney U-test was used for continuous variables. All statistical analyses were performed using IBM SPSS Statistics ver. 23.0 (IBM Co., Armonk, NY, USA). A P-value of $<0.05$ was considered to be statistically significant.

\section{RESULTS}

\section{Pre- and Perioperative Patient Characteristics}

Of the 18 patients that were eligible to participate in the study, two died and one was lost-to-follow-up. Table 1 shows baseline 
Table 1. Preoperative patient characteristics of 15 patients with available follow-up data that underwent further analysis in the current case series

\begin{tabular}{lc}
\hline Characteristic & Value \\
\hline Age $(\mathrm{yr})$ & $69.8 \pm 8.2$ \\
Mean \pm SD & $71.1(53.3-85.1)$ \\
Median (range) & \\
Body mass index $\left(\mathrm{kg} / \mathrm{m}^{2}\right)$ & $27.6 \pm 3.1$ \\
Mean \pm SD & $27.2(23.0-33.1)$ \\
Median (range) & \\
Duration of incontinence (mo) & $41.1 \pm 41.3$ \\
Mean \pm SD & $20(3.0-131.0)$ \\
Median (range) & \\
Daily pad usage & $4.6 \pm 1.5$ \\
Mean \pm SD & $4.5(2.0-7.0)$ \\
Median (range) & \\
IQoL score & $37.3 \pm 16.6$ \\
Mean \pm SD & $31.8(15.9-68.2)$ \\
Median (range) & \\
ICIQ-SF score & $14.1 \pm 5.2$ \\
Mean \pm SD & $15.0(4.0-21.0)$ \\
Median (range) &
\end{tabular}

SD, standard deviation; ICIQ-SF, International Consultation on Incontinence Questionnaire Short Form; IQoL, International Quality of Life score.

characteristics of the 15 remaining consecutive patients that were included in the study and underwent further analysis. Eight patients (53.3\%) suffered from cardiovascular disease and 2 patients (13.3\%) suffered from diabetes mellitus.

\section{Efficacy After AdVance and AdVanceXP Male Sling Implantation}

Median follow-up was 70 months (range, 18-83 months; mean standard \pm deviation: $62 \pm 21$ months). In detail, median followup after AdVance implantation was 72 months, and median follow-up after AdVanceXP implantation was 36 months $(\mathrm{P}=0.03)$.

Notably, 2 out of 15 patients (13.3\%) had an artificial urinary sphincter implanted during the follow-up period. Regarding success rates, these patients were classified as treatment failure. They were excluded from further pad use and pad test analysis. The descriptive efficacy outcome of 15 patients after a combined median follow-up of nearly 6 years is summarized in Table 2. A detailed analysis of the efficacy outcome of each individual patient that underwent further analysis in the current case series is given in Table 3. A longitudinal analysis was per
Table 2. Efficacy outcome of 15 patients who underwent further analysis after a median follow-up of 70 months

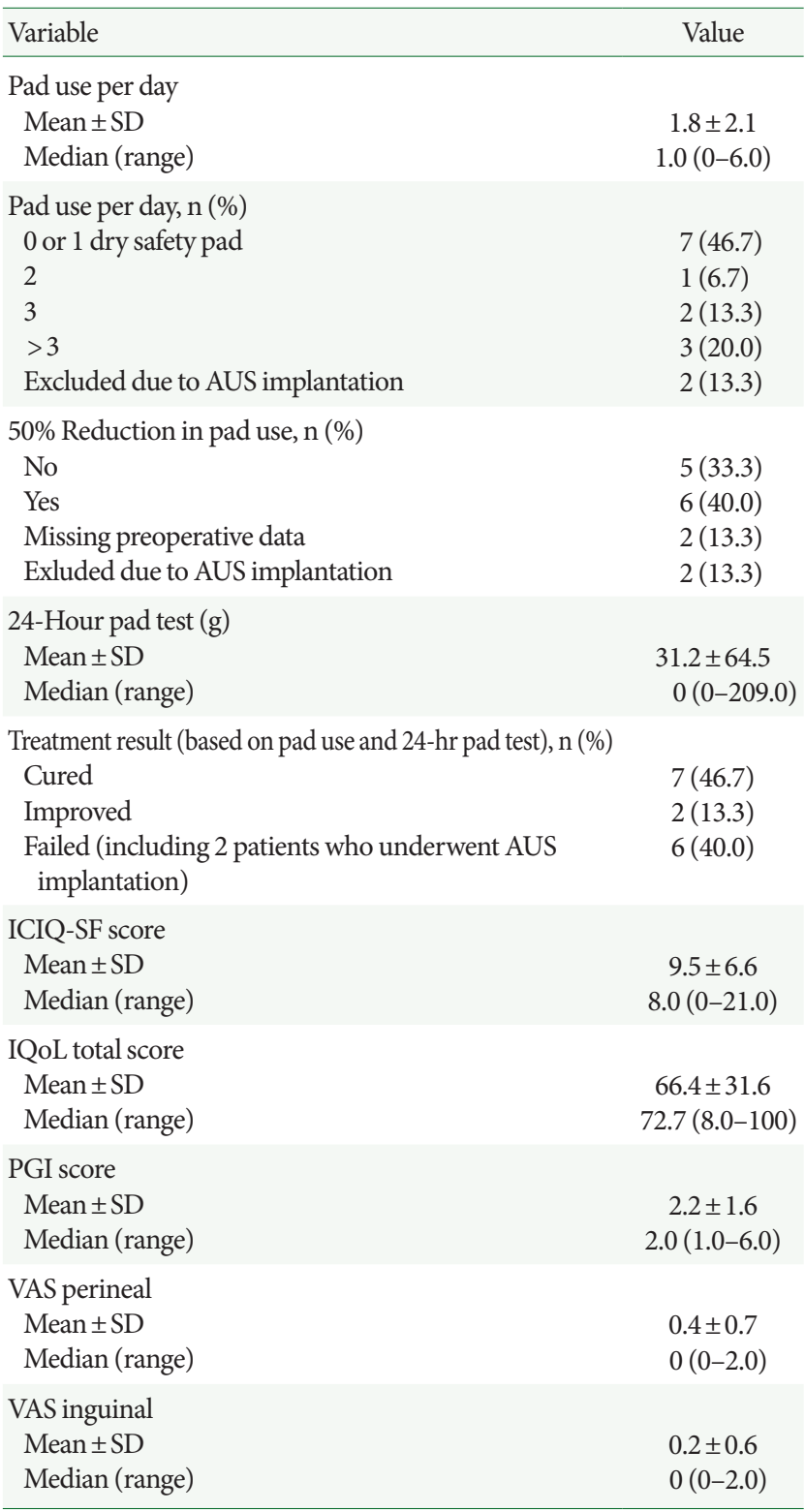

SD, standard deviation; AUS, artificial urinary sphincter; ICIQ-SF, International Consultation on Incontinence Questionnaire Short Form; IQoL, International Quality of Life score; PGI, patient's global impression of improvement score; VAS, visual analogue scale.

formed for the efficacy outcome variables that were available both pre- and postoperatively (Table 4). Daily pad usage decreased significantly $(\mathrm{P}=0.015)$ while quality of life based on mean IQoL scores increased significantly $(\mathrm{P}=0.050)$ over the same period. 


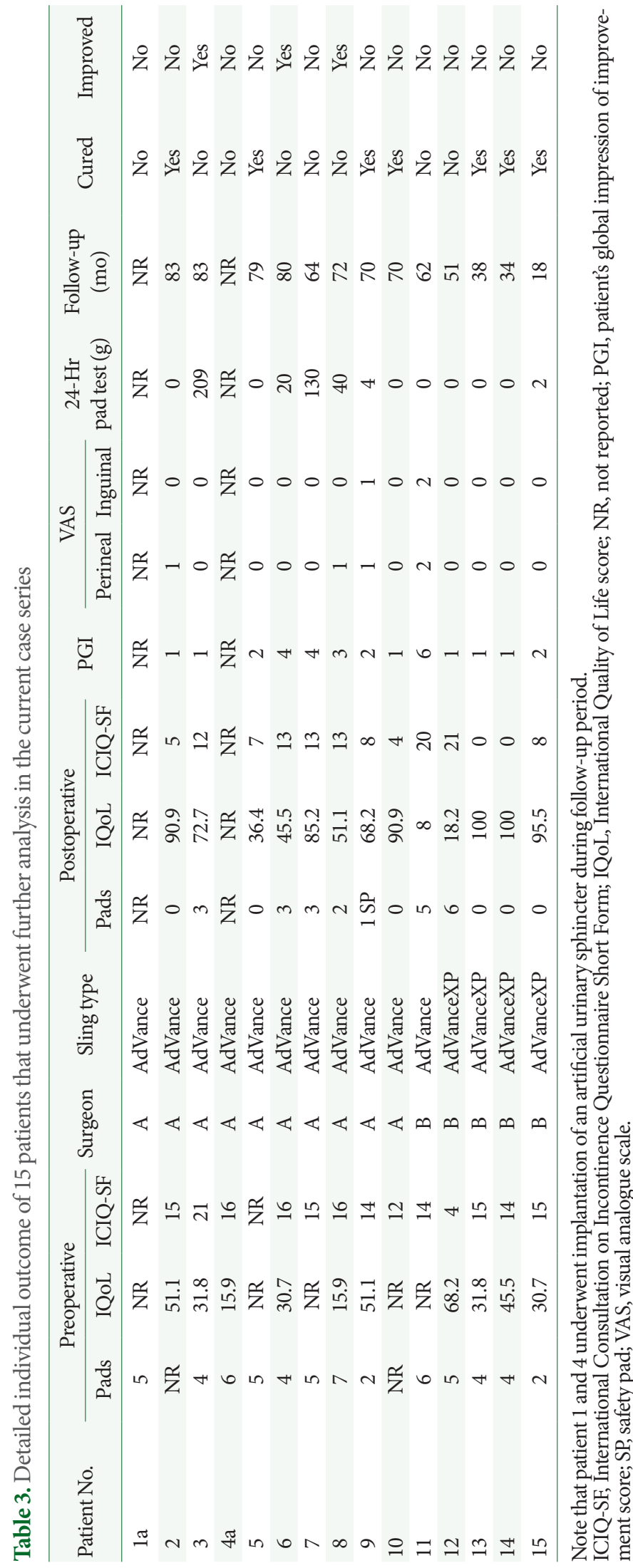

Table 4. Longitudinal analysis of ICIQ-SF, IQOL, and daily pad usage before and after AdVance or AdVanceXP male sling implantation

\begin{tabular}{lcccc}
\hline Variable & Preoperatively & Follow-up & $\Delta$ & P-value \\
\hline Pads per day & $4.6 \pm 1.6$ & $1.8 \pm 2.1$ & -2.8 & $0.015^{*}$ \\
ICIQ-SF & $14.4 \pm 3.7$ & $9.5 \pm 6.6$ & -4.9 & 0.077 \\
IQoL & $37.3 \pm 16.6$ & $66.4 \pm 31.6$ & 29.1 & $0.050^{*}$ \\
\hline
\end{tabular}

Values are presented as mean \pm standard deviation.

ICIQ-SF, International Consultation on Incontinence Questionnaire Short Form; IQoL, International Quality of Life score.

${ }^{\star} \mathrm{P}<0.05$.

Table 5. Analysis of various hypothesized predictive features for success after AdVance or AdVanceXP male sling implantation based on the outcome of 15 patients that underwent further analysis in the current case series

\begin{tabular}{lll}
\hline Predictive feature & Success rate $(\%)$ & P-value \\
\hline $\begin{array}{l}\text { Preoperative pad usage } \\
\leq 5 \text { per day, yes vs. no }\end{array}$ & 62.5 vs. 0 & 0.182 \\
$\quad \leq 4$ per day, yes vs. no & 83.3 vs. 22.2 & $0.041^{*}$ \\
$\begin{array}{l}\text { Age } \leq 71 \text { yr } \\
\quad \text { Yes vs. no }\end{array}$ & 75.0 vs. 14.1 & $0.041^{*}$ \\
$\begin{array}{l}\text { Body mass index } \leq 27 \mathrm{~kg} / \mathrm{m}^{2} \\
\quad \text { Yes vs. no }\end{array}$ & 71.4 vs. 25.0 & 0.132 \\
$\begin{array}{l}\text { Cardiovascular disease } \\
\text { Yes vs. no }\end{array}$ & 28.6 vs. 62.5 & 0.315 \\
$\begin{array}{l}\text { Surgeon } \\
\text { A vs. B }\end{array}$ & 40.0 vs. 60.0 & 0.608 \\
Sling type & & \\
AdVance vs. AdVanceXP & 36.4 vs. 75.0 & 0.282 \\
\hline
\end{tabular}

\section{Predictive Features for Success After AdVance or AdVanceXP Implantation After TURP}

Potential associative factors that were hypothesized to contribute to each individual patient's success were analyzed. To address the impact of age, patients were divided into 2 groups ( $\leq 71$ years vs. $>71$ years) based on the median age of our patient cohort. To analyze the impact of preoperative incontinence based on preoperative daily pad usage, we performed two separate analyses ( $\leq 4$ pads vs. $>4$ pads; $\leq 5$ pads vs. $>5$ pads) following the median preoperative daily pad usage of our patient cohort (4 pads), as well as a definition of moderate stress urinary continence (5 pads) that has been frequently used for outcome analyses of retrourethral transobturator male slings $[6,10,22]$.

Results of the univariate analysis are summarized in Table 5. 
Briefly, significantly better outcomes were found for patients needing less than 5 pads per day preoperatively $(\mathrm{P}=0.041)$ as well as for patients with a preoperative age of $\leq 71$ years (based on median age of our patient cohort; $\mathrm{P}=0.041$ ) at the time of implantation. Preoperative pad usage and age were also analyzed as continuous variables using the Mann-Whitney U-test. Hereby, the significant impact of preoperative pad usage $(\mathrm{P}=$ 0.009) could be confirmed, while no significant results could be observed for patient age $(\mathrm{P}=0.463)$.

\section{Safety}

There was one intraoperative complication (5.6\%, urethral perforation; Clavien-Dindo grade IIIa) during AdVance implantation. Acute urinary retention occurred in 3 patients (16.7\%; Clavien-Dindo grade II) after removal of the transurethral Foley catheter 4 days postoperation. A new transurethral catheter was placed in one patient and could be removed after 2 more days. Two patients were instructed to perform clean intermittent self-catheterization that could be discontinued 2 weeks postoperatively in both cases without any further therapy. There were no further complications reported. There were no differences in terms of postoperative complication rates between the AdVance and AdVanceXP male sling. According to VAS, inguinal as well as perineal pain was negligible in the long-term follow-up.

\section{DISCUSSION}

The current retrospective single-center study was conducted to address the increasing need for evidence regarding the optimal treatment of post-TURP SUI. A success rate of $38.9 \%$ and a cure-and-improved rate of $50.0 \%$ in the long-term follow-up were found.

The strength of this study is the use of multiple validated tools to determine the efficacy outcome as well as a long followup period. Postoperative incontinence was measured using daily pad usage, 24-hour pad testing and the validated ICIQ-SF. Quality of life was tested by the validated IQoL score and patient satisfaction was determined using the validated PGI score. A postoperative increase of patients' quality of life in the longterm follow-up was found that reached statistical significance in the longitudinal analysis.

In the current study, several potential associative factors were assessed that might contribute to increased success rates. Significantly better outcomes were found for patients with a pre- operative age of $\leq 71$ years. This cutoff value was chosen based on the median age of the patient cohort. Thus, the impact of patient's age was analyzed continuously and the statistical significance could not be confirmed. It has to be stated that the results might indicate a more favorable outcome of the AdVance or AdVanceXP male sling in younger patients with SUI after TUR-P but this hypothesis has to be proven in larger patient cohorts.

Significantly higher success rates were found in patients who needed 4 or fewer pads per day before the implantation. This cutoff value is based on the median preoperative pad usage of our patient cohort. Since the use of retrourethral transobturator male slings is generally only recommended in patients with mild-to-moderate SUI, another cutoff value was tested for that was based on current frequently used definitions of moderate male SUI $[3,6,10,22]$. No statistically significant impact could be observed while using the latter cutoff value (fewer than 6 pads per day). These results have to be interpreted with caution considering the small sample size of the current case series. This study's results are in line with the findings of Collado Serra et al. [23] who previously reported that the results of retrourethral transobturator male slings may be inversely related to the preoperative severity of incontinence after radical prostatectomy.

Several studies have evaluated the outcome after AdVance implantation, and there is currently data up to a maximum follow-up of 39 months available $[4,6,7,9-11,24,25]$. However, none of the aforementioned studies focused on post-TURP SUI. Regarding PPI, the longest follow-up after AdVanceXP implantation is 2 years in a prospective multicenter study, and nearly 3 years in a recent prospective single-center study $[8,20]$. Thus, the current study provides the longest follow-up after retrourethral transobturator male sling implantation to date. Regarding the long-term outcome after AdVance male sling implantation, Rehder et al. [9] recently published the outcome after a mean follow-up of 3 years. The authors reported a curedand-improved rate of $76.8 \%$. However, in the study by Rehder et al. [9], definition of success was based on daily pad usage (no pad or one dry safety pad) only. Zuckerman et al. [25] retrospectively analyzed 102 patients with PPI and found a 3-year cure rate (no pad or one dry safety pad) of $42 \%$ and a cure-andimproved rate of $62 \%$. Wadie [11] studied the outcome of a retropubic bulbourethral male sling that was designed at Mansoura University and is similar to the AdVance male sling and found a dry rate of $85 \%$ (based on daily pad usage) after 2 years 
of follow-up. Notably, the author included 17 patients following TURP but did not report a separate subgroup analysis. In addition, there is also evidence from studies that used stricter definitions of 'cure.' A recent prospective multicenter study that includes data from 94 consecutive patients after AdVanceXP implantation and a maximum follow-up of 2 years, showed a cure rate (defined as $\leq 5 \mathrm{~g}$ in a 24-hour pad test and no pads used) of $73.1 \%$. Cure-and-improved rate was $92.7 \%$ [8]. In summary, one can state that the success rate of the current study is lower than the reported cure rates that focus on the treatment of SUI after radical prostatectomy.

This might be explained by a different pathophysiology determining SUI after TURP and after radical prostatectomy. It has been suggested that the method of action of retrourethral transobturator male slings after radical prostatectomy is based on the repositioning of the posterior urethra, a correction of urethral hypermobility, and a venous sealing effect. However, after TURP, the dislocation of the posterior urethra and consecutive urethral hypermobility is supposedly less weighty. The venous sealing effect, on the other hand, is probably equally evident in the post-TURP setting. To obtain good continence results after AdVance or AdVanceXP sling implantation, an unimpaired residual sphincter function is crucial. External urethral sphincter lesions are more common after TURP and can be caused during the resection itself as well as through constant pressure of the resectoscope. These lesions are usually visible during the repositioning test and disqualify the patient for AdVance or AdVanceXP implantation [14]. However, one might speculate that minor sphincter lesions that cannot be detected during preoperative urethroscopy contribute to the unfavorable long-term outcome after TURP as presented in the current study.

Lastly, these results indicate that complication rates of AdVance and AdVanceXP male sling implantation are comparable after TURP and radical prostatectomy. Notably, there were no Clavien-Dindo grades IV and V complications with acute urinary retention being the most frequent complication.

The current study is not devoid of limitations. First and foremost are the limitations inherent to retrospective analyses. The caseload of our study is small compared to current studies investigating the outcome of the AdVance and AdVanceXP male sling in the treatment of PPI. However, to our knowledge, this is the first study presenting results particularly after TURP. The presented cohort includes patients after AdVance as well as after AdVanceXP implantation. However, there are currently two comparative studies available that show that the efficacy of the AdVanceXP sling was not significantly different compared to the conventional AdVance sling [19,22]. Despite its limitations, the findings of the current study may have an impact on current clinical practice.

To summarize, the current study is the first to provide a longterm follow-up of 70 months after retrourethral transobturator male sling implantation. It is able to show that successful treatment of TURP-induced SUI with the AdVance or AdVanceXP male sling is possible, but success rates seem to be lower than for the treatment of SUI after radical prostatectomy and patients have to be counseled accordingly. Furthermore, this study shows that AdVance and AdVanceXP male slings can be safely implanted after TURP. Finally, it is shown that patients with a preoperative age of $\leq 71$ years who need up to 4 pads preoperatively might benefit the most from AdVance or AdVanceXP implantation after TURP.

\section{REFERENCES}

1. Lucas MG, Bosch RJ, Burkhard FC, Cruz F, Madden TB, Nambiar AK, et al. EAU guidelines on surgical treatment of urinary incontinence. Eur Urol 2012;62:1118-29.

2. Kumar A, Litt ER, Ballert KN, Nitti VW. Artificial urinary sphincter versus male sling for post-prostatectomy incontinence--what do patients choose? J Urol 2009;181:1231-5.

3. Kretschmer A, Hübner W, Sandhu JS, Bauer RM. Evaluation and management of postprostatectomy incontinence: a systematic review of current literature. Eur Urol Focus 2016;2:245-59.

4. Bauer RM, Mayer ME, Gratzke C, Soljanik I, Buchner A, Bastian $\mathrm{PJ}$, et al. Prospective evaluation of the functional sling suspension for male postprostatectomy stress urinary incontinence: results after 1 year. Eur Urol 2009;56:928-33.

5. Bauer RM, Soljanik I, Füllhase C, Karl A, Becker A, Stief CG, et al. Mid-term results for the retroluminar transobturator sling suspension for stress urinary incontinence after prostatectomy. BJU Int 2011;108:94-8

6. Cornu JN, Sèbe P, Ciofu C, Peyrat L, Cussenot O, Haab F. Midterm evaluation of the transobturator male sling for post-prostatectomy incontinence: focus on prognostic factors. BJU Int 2011;108: 236-40.

7. Kowalik CG, DeLong JM, Mourtzinos AP. The advance transobturator male sling for post-prostatectomy incontinence: subjective and objective outcomes with 3 years follow up. Neurourol Urodyn 2015;34:251-4. 
8. Bauer RM, Gozzi C, Klehr B, Kretschmer A, Grabbert M, Rehder P, et al. AdVanceXP male sling: 2-year results of a multicentre study. World J Urol 2016;34:1025-30.

9. Rehder P, Haab F, Cornu JN, Gozzi C, Bauer RM. Treatment of postprostatectomy male urinary incontinence with the transobturator retroluminal repositioning sling suspension: 3-year followup. Eur Urol 2012;62:140-5.

10. Cornu JN, Sèbe P, Ciofu C, Peyrat L, Beley S, Tligui M, et al. The AdVance transobturator male sling for postprostatectomy incontinence: clinical results of a prospective evaluation after a minimum follow-up of 6 months. Eur Urol 2009;56:923-7.

11. Wadie BS. Retropubic bulbourethral sling for post-prostatectomy male incontinence: 2-year followup. J Urol 2010;184:2446-51.

12. Gratzke C, Bachmann A, Descazeaud A, Drake MJ, Madersbacher S, Mamoulakis C, et al. EAU Guidelines on the assessment of nonneurogenic male lower urinary tract symptoms including benign prostatic obstruction. Eur Urol 2015;67:1099-109.

13. Bauer RM, Hampel C, Haferkamp A, Höfner K, Hübner W; Für den Arbeitskreis „Urologische Funktionsdiagnostik und Urologie der Frau“ der Akademie der Deutschen Gesellschaft für Urologie. Diagnosis and surgical treatment of postprostatectomy stress incontinence: recommendation of the working group Urologische Funktionsdiagnostik und Urologie der Frau. Urologe A 2014;53: 847-53.

14. Bauer RM, Gozzi C, Roosen A, Khoder W, Trottmann M, Waidelich R, et al. Impact of the 'repositioning test' on postoperative outcome of retroluminar transobturator male sling implantation. Urol Int 2013;90:334-8.

15. Rehder P, Gozzi C. Transobturator sling suspension for male urinary incontinence including post-radical prostatectomy. Eur Urol 2007;52:860-6.

16. Avery K, Donovan J, Peters TJ, Shaw C, Gotoh M, Abrams P. ICIQ: a brief and robust measure for evaluating the symptoms and impact of urinary incontinence. Neurourol Urodyn 2004;23:322-30.
17. Bushnell DM, Martin ML, Summers KH, Svihra J, Lionis C, Patrick DL. Quality of life of women with urinary incontinence: cross-cultural performance of 15 language versions of the I-QOL. Qual Life Res 2005;14:1901-13.

18. Twiss CO, Fischer MC, Nitti VW. Comparison between reduction in 24-hour pad weight, International Consultation on Incontinence-Short Form (ICIQ-SF) score, International Prostate Symptom Score (IPSS), and Post-Operative Patient Global Impression of Improvement (PGI-I) score in patient evaluation after male perineal sling. Neurourol Urodyn 2007;26:8-13.

19. Bauer RM, Kretschmer A, Stief CG, Füllhase C. AdVance and AdVance XP slings for the treatment of post-prostatectomy incontinence. World J Urol 2015;33:145-50.

20. Kretschmer A, Grabbert M, Sommer A, Stief CG, Bauer RM. Midterm outcomes after AdVanceXP male sling implantation. BJU Int 2016;118:458-63.

21. Dindo D, Demartines N, Clavien PA. Classification of surgical complications: a new proposal with evaluation in a cohort of 6336 patients and results of a survey. Ann Surg 2004;240:205-13.

22. Cornu JN, Batista Da Costa J, Henry N, Peyrat L, Beley S, Haab F. Comparative study of AdVance and AdVanceXP male slings in a tertiary reference center. Eur Urol 2014;65:502-4.

23. Collado Serra A, Resel Folkersma L, Domínguez-Escrig JL, Gómez-Ferrer A, Rubio-Briones J, Solsona Narbón E. AdVance/AdVance XP transobturator male slings: preoperative degree of incontinence as predictor of surgical outcome. Urology 2013;81:1034-9.

24. Rehder P, Mitterberger MJ, Pichler R, Kerschbaumer A, Glodny B. The 1 year outcome of the transobturator retroluminal repositioning sling in the treatment of male stress urinary incontinence. BJU Int 2010;106:1668-72.

25. Zuckerman JM, Edwards B, Henderson K, Beydoun HA, McCammon KA. Extended outcomes in the treatment of male stress urinary incontinence with a transobturator sling. Urology 2014;83: 939-45. 